\title{
Antibodies specific to Ferritin, light polypeptide (FTL) are frequently detected in Immunorelated Pancytopenia patients
}

\author{
Shanfeng $\mathrm{Hao}^{1}$, yang zhang ${ }^{1}$, luogang hua ${ }^{1}$, ning xie $^{1}$, na xiao ${ }^{1}$, xiaojing $\mathrm{wu}^{2}$, huaquan \\ wang ${ }^{1}$, rong $\mathrm{fu}^{1}$, and zonghong shao ${ }^{1}$ \\ ${ }^{1}$ Tianjin Medical University General Hospital \\ ${ }^{2}$ Peking University BinHai Hospital
}

May 5, 2020

\begin{abstract}
Immuno-related pancytopenia (IRP) is featured by pancytopenia resulting from bone marrow suppression or destruction mediated by autoantibodies (auto-Abs). In a previous study we have established a K562 cDNA library, which was applied in screening 7 possible autoantigens produced by hematopoietic cells in IRP patients, including FTL. In this study FTL was expressed and purified, and then we detected the level of the auto-Abs specific to FTL. Through enzyme-linked immunosorbent assay (ELISA), it is revealed that the titer of anti-FTL antibodies (FTL Abs) was higher in IRP patients without treatment compared with those who recovered from IRP, those with severe aplastic anemia(SAA), those with myelodyplastic syndrome(MDS) and healthy controls. Furthermore, the level of FTL-mRNA was up-regulated in IRP patients without treatment compared with those who recovered from IRP, MDS patients and normal controls. These findings indicate that FTL Abs are highly expressed in IRP patients. Detecting FTL Abs might have some clinical value in differentiating IRP from SAA and MDS. Moreover, in certain type of IRP patients, FTL, as an autoantigen, may induce immune attack on hematopoietic stem cells.
\end{abstract}

\section{Hosted file}

Figure 1.tif available at https://authorea.com/users/297598/articles/426791-antibodiesspecific-to-ferritin-light-polypeptide-ftl-are-frequently-detected-in-immunorelatedpancytopenia-patients 